\title{
Entalhamento de cerâmicas para medida de tenacidade à fratura pelo método SEVNB
}

\section{(Notching of ceramics as preparation of specimens for fracture toughness measurements using the SEVNB method)}

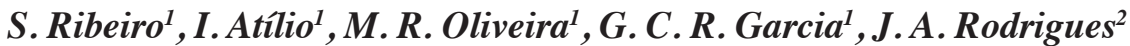 \\ ${ }^{I}$ Departamento de Engenharia de Materiais - DEMAR, Escola de Engenharia de Lorena - EEL, Universidade de \\ S. Paulo - USP, Estrada Santa Lucrecia s/n, Bairro Mondezir, CP 116, Lorena SP 12600-970 \\ ${ }^{2}$ Departamento de Engenharia de Materiais - DEMa, Universidade Federal de S. Carlos - UFSCar \\ sebastião@demar.eel.usp.br
}

\begin{abstract}
Resumo
O objetivo deste trabalho é apresentar uma máquina para entalhamento de corpos de prova cerâmicos, bem como a escolha e a execução do entalhamento, usando $\mathrm{SiC}$ sinterizado via fase líquida, com adições de $\mathrm{Al}_{2} \mathrm{O}_{3}$ e $\mathrm{Yb}_{2} \mathrm{O}_{3}$, como material experimental para ser entalhado. Foi desenvolvido e construído, junto a uma empresa de S. Carlos-SP, um equipamento que permite produzir entalhes finos em corpos cerâmicos para posterior ruptura, visando determinar $\mathrm{K}_{\mathrm{IC}}$ pelo método SEVNB. Amostras de SiC com $10 \%$ de $\mathrm{Al}_{2} \mathrm{O}_{3}+\mathrm{Yb}_{2} \mathrm{O}_{3}$ foram sinterizadas a $1950{ }^{\circ} \mathrm{C}$, retificadas e entalhadas com lâminas de barbear e pastas diamantadas de $15,9,6,3$, 1 e $0,25 \mu \mathrm{m}$. A máquina produzida para o entalhamento das amostras está em operação no DEMAR-EEL-USP e, ao que se sabe, é a primeira no Brasil. Os resultados mostraram que em função da espessura da lâmina e da granulometria do agente abrasivo, pode-se produzir entalhes com diferentes raios de curvatura de sua ponta e profundidades.
\end{abstract}

Palavras-chave: tenacidade à fratura, SEVNB, entalhamento, SiC.

\begin{abstract}
The aim of this work is to present a machine to produce notches in ceramic bodies as well the choice and how to make the notches. $\mathrm{SiC}$-ceramics was produced by liquid phase sintering as testing material. For the liquid sintering, a mixture of $\mathrm{Al}_{2} \mathrm{O}_{3}$ and $\mathrm{Yb}_{2} \mathrm{O}_{3}$ as additives was applied. The machine was developed and built by a company sited in S. Carlos-SP. It permits to obtain narrow notches in ceramic specimens to be fractured afterwards. That is to facilitate the measurement of $K_{I C}$ based on the SEVNB method. Specimens of $10 \%$ of $\left(\mathrm{Al}_{2} \mathrm{O}_{3}+\mathrm{Yb}_{2} \mathrm{O}_{3}\right)$ containing $\mathrm{SiC}$ were sintered at $1950{ }^{\circ} \mathrm{C}$. Those specimens were machined and notched using the machine and razor blades and diamond pastes of 15, 9, 6, 3, 1 and $0.25 \mu$ m of particle size. The built machine is installed at DEMAR-EEL-USP, and it is said to be the first of that type in Brazil. The results showed that depending on the thickness of the razor blade and the size of the diamond particles, it can be produced notches with distinct tip radius and notch depth values.
\end{abstract}

Keywords: fracture toughness, SEVNB, notching, SiC.

\section{INTRODUÇÃO}

Cerâmicas são materiais de elevada importância em muitas aplicações tecnológicas, principalmente nos trabalhos onde se exige alto desempenho. Possuem elevada resistência mecânica e química mesmo em condições de carregamento extremo. Infelizmente, uma grande desvantagem das cerâmicas é a baixa tenacidade à fratura, $\mathrm{K}_{\mathrm{IC}}$, que as tornam motivo de preocupação em aplicações que exigem altos valores dessa propriedade [1-3].

A tenacidade à fratura é uma característica importante das cerâmicas, que mede a resistência ao início de propagação de trincas. Entretanto, a medida dessa propriedade é ainda motivo de discussões e controvérsias, existindo vários métodos para a sua determinação, uns padronizados e outros não, além de diferentes equações para seu cálculo.
Os principais métodos para a determinação da tenacidade à fratura são: IF (Indentation Fracture) [2, 4-8], IS (Indentation Strength) [5, 9], CNB (Chevron Notch Beam) [3, 10-12], SCF (Surface Crack in Flexure) $[9,11,12]$, SEPB (Single Edge Precracked Beam) [2, 3, 9, 11, 13], SENB (Single Edge Notched Beam) $[10,12,13]$, SEVNB (Single Edge V-Notched Beam) [1, 3-7, 9, 11, 13-15]. A Fig. 1 ilustra os corpos de prova e os tipos de defeitos artificiais introduzidos nos mesmos para a medida de $\mathrm{K}_{\mathrm{IC}}$. As siglas em inglês foram mantidas por serem amplamente conhecidas dessa forma.

Os métodos IF e IS são baseados em constantes empíricas para determinar $\mathrm{K}_{\mathrm{IC}}$ e são menos rigorosos sob o ponto de vista teórico da mecânica da fratura que os outros métodos $[9,16]$. O método SEVNB, que consiste na quebra por flexão de uma barra entalhada em "V", é uma versão mais elaborada do método SENB, que já foi um dos 
métodos com maior aceitabilidade na medida de $\mathrm{K}_{\mathrm{IC}}$ [6].

Há algumas décadas que esforços foram envidados no sentido de se aprimorar o método SEVNB, mas só em 2008 foi editada uma norma que estabelece as diretrizes de execução do método, a norma ISO 23146:2008 [14]. Mesmo assim, ainda continua a discussão da aceitabilidade dessa norma para cerâmicas tenacificadas, como é o caso das cerâmicas à base de zircônia. A própria norma não aconselha sua utilização para tais cerâmicas [14].

Além do exposto, é muito discutida na literatura a influência do raio de curvatura da ponta do entalhe, simbolizado por $\mathrm{R}$, pois a partir de um tamanho crítico as medidas de tenacidade à fratura são superestimadas [2, $3,8,14,17,18]$. Esse fato pode ser visto com clareza na Fig. 2, pois no ponto em que $\mathrm{K}$ passa a ser dependente de $R$, tem-se o raio crítico, $R_{C}$, e daí para frente $K_{I C}$ cresce linearmente com a raiz quadrada de R. O gráfico da Fig. 2a é hipotético, mas o $2 \mathrm{~b}$ foi montado com dados experimentais para uma cerâmica de $\mathrm{ZrO}_{2}$, sendo a curva mais escura também calculada [18].

Não só o raio de curvatura da ponta do entalhe é importante na determinação de $\mathrm{K}_{\mathrm{IC}}$ pelo método SEVNB, mas também a microestrutura da cerâmica. Foi provado empiricamente que o valor de tenacidade pode ser considerado verdadeiro se o raio de curvatura da ponta do entalhe é menor que duas vezes o tamanho médio de grão do material [19], confirmando as mesmas conclusões reportadas $[19,20]$.

Matematicamente a relação entre $\mathrm{K}_{\mathrm{IC}}$, raio de curvatura da ponta do entalhe, e tamanho de grão, pode ser expressa como:

$$
\begin{array}{ll}
\mathrm{K}_{\mathrm{b}}=\mathrm{K}_{\mathrm{IC}} & \mathrm{R} \leq 2 \mathrm{~d} \\
\mathrm{~K}_{\mathrm{b}}=\mathrm{K}_{\mathrm{IC}}+\mathrm{m}(\mathrm{R}-2 \mathrm{~d})^{1 / 2} & \mathrm{R}>2 \mathrm{~d}
\end{array}
$$

emque $\mathrm{K}_{\mathrm{b}}$ éatenacidadeàfraturamedidaexperimentalmente, $\mathrm{K}_{\mathrm{IC}}$ é o valor real da tenacidade à fratura, $\mathrm{R}$ é o raio de curvatura da ponta do entalhe e d é o tamanho médio de grão. A inclinação da parte retilínea do gráfico de $\mathrm{K}_{\mathrm{b}} \mathrm{x} \mathrm{d}^{1 / 2}$, $\mathrm{m}$, pode ser interpretada como a sensibilidade do material a superestimação da tenacidade à fratura [17].

Fazendo uma avaliação detalhada da literatura, percebe-se que o método SEVNB é o que menos apresenta problemas, tanto na medição quanto no cálculo de $\mathrm{K}_{\mathrm{IC}}$, podendo ser aplicado com sucesso, principalmente quando se dispõe de uma máquina adequada para a execução do entalhe fino, por exemplo, usando uma lâmina de barbear $[1,4,18]$. O método SEVNB não é difundido e aplicado no Brasil, pois são preferidos os mais simples, mesmo sabendo que não são tão precisos e confiáveis. Logo, espera-se que este trabalho venha contribuir para a divulgação e aceitação dessa técnica para medida de $\mathrm{K}_{\mathrm{IC}}$ de cerâmicas avançadas no Brasil, pois o entalhamento não é mais o problema, além de que fazer flexão em quatro pontos é trivial e amplamente conhecido.

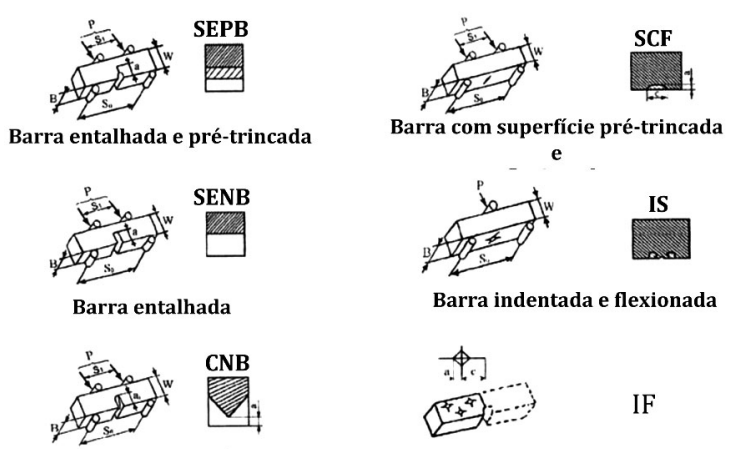

Barra entalhada "chevron"

Barra fraturada por indentação

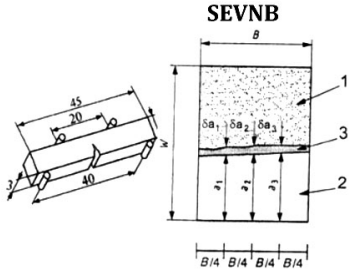

Barra entalhada em "V"

Figura 1: Principais métodos para determinação da tenacidade à fratura. Figura adaptada de [11].

[Figure 1: Main methods to measure toughness fracture. Figure adapted from [11].]
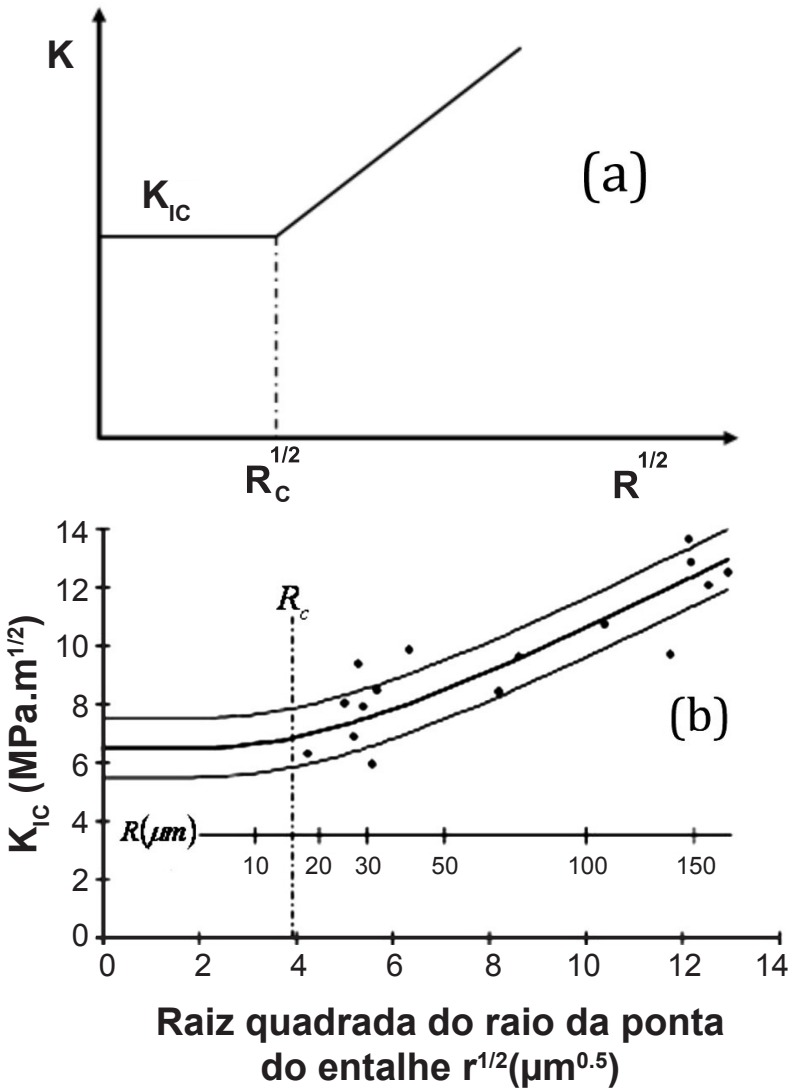

Figura 2: Representação esquemática da dependência de $\mathrm{K}_{\mathrm{IC}}$ com a raiz quadrada do raio de curvatura da ponta do entalhe, $\mathrm{R}^{1 / 2}$, adaptado de [2] e [18].

[Figure 2: Schematic representation for the $K_{I C}$ dependence on the square root of the radius of the notch tip, $R^{1 / 2}$. Adapted from [2] and [18].] 


\section{MATERIAIS E MÉTODOS}

\section{Materiais}

\subsection{Barras de SiC sinterizadas via fase líquida}

O material de teste usado neste trabalho foi uma cerâmica de $\mathrm{SiC}$, contendo $10 \%$ em volume de uma mistura eutética de $\mathrm{Al}_{2} \mathrm{O}_{3}-\mathrm{Yb}_{2} \mathrm{O}_{3}$, prensada isostaticamente a frio e sinterizada via fase líquida a $1950{ }^{\circ} \mathrm{C}$ por $1 \mathrm{~h}$, em atmosfera de argônio. A densidade relativa desse material é de 90\%. A Fig. 3 mostra a microestrutura do $\mathrm{SiC}$ usado, obtida por microscopia eletrônica de varredura, MEV, no modo elétrons secundários.

As barras de $\mathrm{SiC}$ sinterizadas foram retificadas com

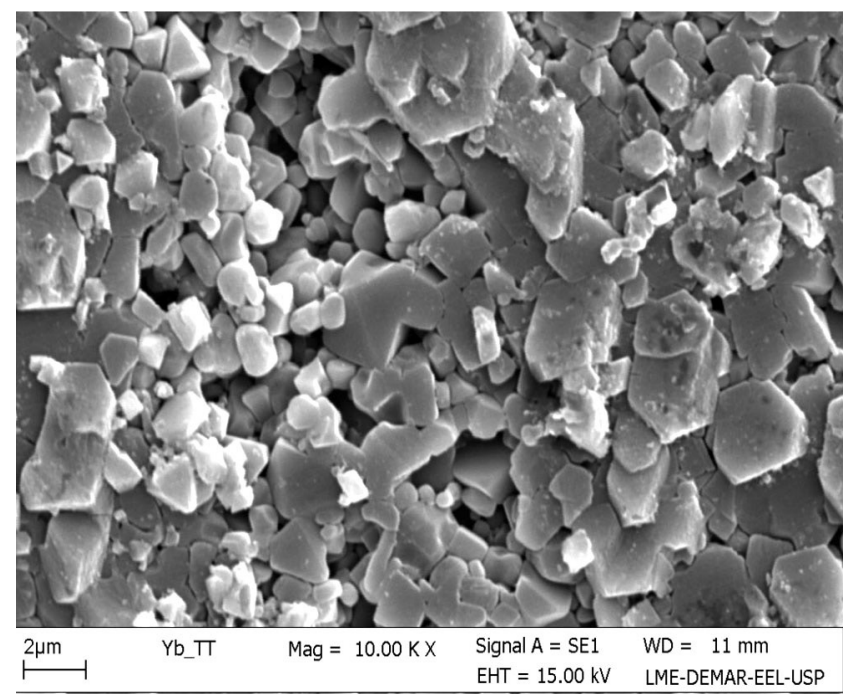

Figura 3: Imagem de $\mathrm{MEV}$, no modo elétrons secundários, da superfície de uma amostra de $\mathrm{SiC}+10 \%\left(\mathrm{Al}_{2} \mathrm{O}_{3}+\mathrm{Yb}_{2} \mathrm{O}_{3}\right)$ usada neste trabalho.

[Figure 3: SEM image, secondary electron mode, of the surface of a $\mathrm{SiC}+10 \%\left(\mathrm{Al}_{2} \mathrm{O}_{3}+\mathrm{Yb}_{2} \mathrm{O}_{3}\right)$ specimen used in this work.]
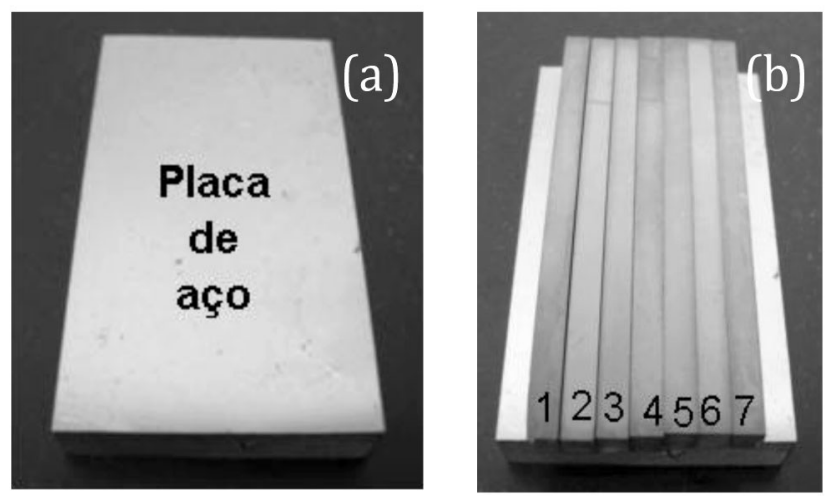

Figura 4: (a) Placa de aço carbono como suporte para as amostras de $\mathrm{SiC}$; e (b) conjunto placa/amostras montadas para acabamento final (passo na retificadora para nivelar todas as amostras) e entalhamento.

[Figure 4: (a) Steel plate to support the SiC specimens and (b) specimens glued on the steel plate to final finishing (step to finish the surface of the specimens) and notching.] rebolo diamantado D121, produzido pela "Winter", até dimensões de $3 \mathrm{~mm}$ x $4 \mathrm{~mm}$ x $50 \mathrm{~mm}$. Após retificação, as amostras foram entalhadas em uma máquina especial para tal função descrita mais adiante.

A Fig. 4 mostra o conjunto amostra/suporte para execução do entalhamento. Foram coladas 7 amostras no suporte, juntadas paralelamente, das quais somente 5 são usadas para o teste de flexão para a determinação do valor de $\mathrm{K}_{\mathrm{IC}}$, conforme norma ISO 23146:2008. As amostras das extremidades são descartadas, pois elas podem apresentar defeitos nas bordas dos entalhes.

1.2. Pastas de diamante nas granulometrias: 0,$25 ; 1$, 3, 6, 9 e 15 um, fornecidas pela Arotec

1.3. Lâminas de barbear nas espessuras de 0,06 $\mathrm{mm}$ (lâmina fina) e 0,21 mm (lâmina grossa)

A Fig. 5 ilustra as lâminas de barbear para promover entalhes com diferentes espessuras (abertura do entalhe).

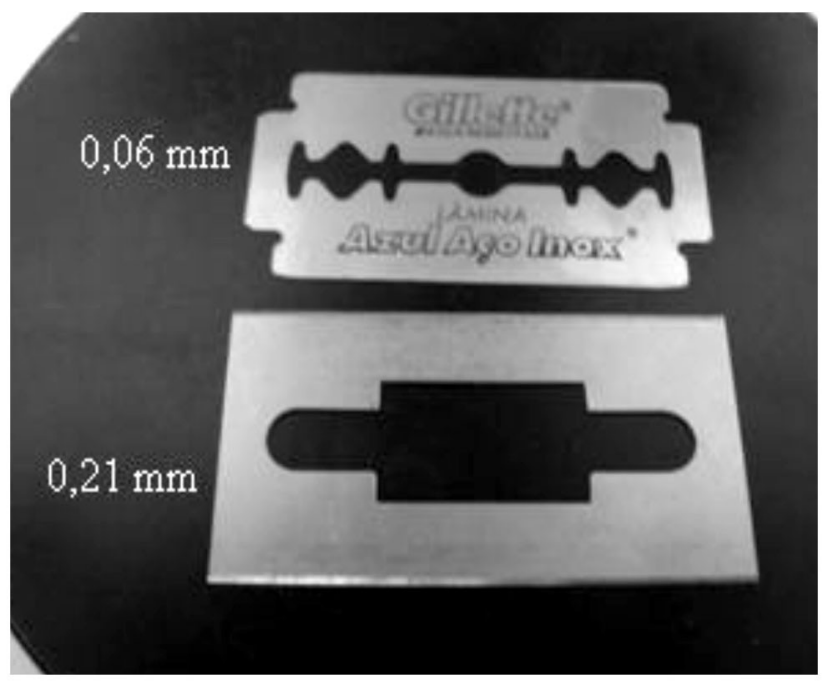

Figura 5: Lâminas de barbear usadas no entalhamento. [Figure 5: Razor blade used to produce notches.]
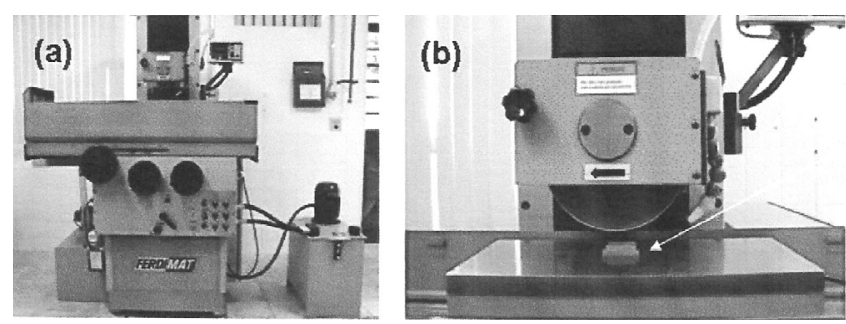

\section{conjunto placa/amostra para retificação e nivelamento final das amostras}

Figura 6: Fotografias da retificadora utilizada para corte e retificação das amostras (a), e detalhe da operação de retificação das amostras (b).

[Figure 6: Pictures of the machine for cutting and grinding of the specimens (a), and detail of the grinding operation to prepare the surface of the specimens.] 


\section{Equipamentos e funcionamento}

\subsection{Retificadora}

A Fig. 6 mostra fotografias da retificadora utilizada para corte, retificação e nivelamento final das amostras para determinação de $\mathrm{K}_{\mathrm{IC}}$ pelo método SEVNB. A Retificadora é do Tipo TA40 da FERDIMAT.

\subsection{Máquina para entalhamento}

A Fig. 7 mostra a máquina desenvolvida para elaboração do entalhe nas amostras, usando lâmina de barbear e pasta de diamante. Essa máquina foi produzida junto a EQUITECS, empresa sediada em S. Carlos-SP, com projeto e adaptações realizadas com suporte do autor S. Ribeiro deste artigo.

Foram realizados testes preliminares com diferentes cargas sobre a lâmina, chegando-se a conclusão de que o próprio peso do sistema que prende a lâmina era suficiente para produzir um entalhe fino e num intervalo de tempo adequado. No caso do SiC, esse tempo está em torno de 2 h. A carga do referido sistema é de $3 \mathrm{~N}$. A velocidade da mesa também foi testada, utilizando-se várias velocidades preliminares e a melhor para as amostras de $\mathrm{SiC}$ foi de 130 deslocações por min.

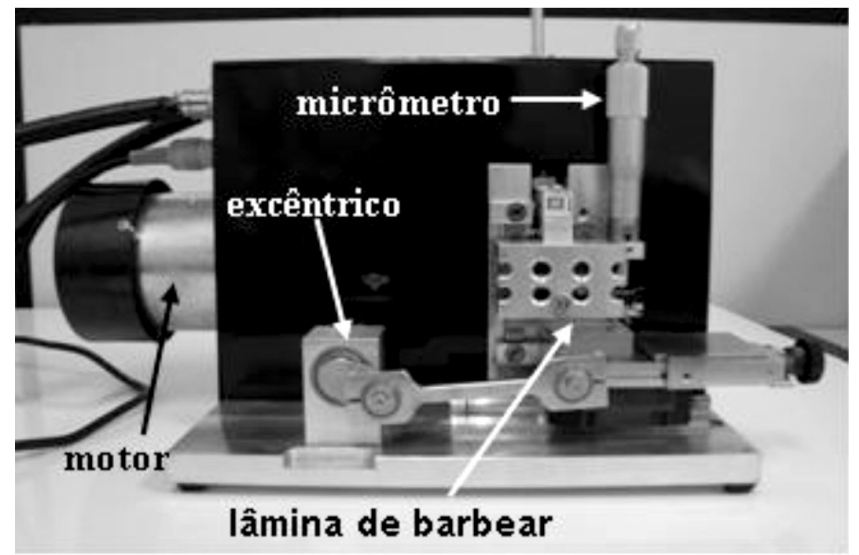

Figura 7: Fotografia da máquina de entalhamento das amostras para a medida de $\mathrm{K}_{\mathrm{IC}}$ pelo método SEVNB.

[Figure 7: Picture of the machine used to notch the specimens to measure the $K_{I C}$ applying the SEVNB method.]

\subsection{Entalhamento}

O entalhamento foi realizado na máquina mostrada na Fig. 7, conforme descrito no item 2.2, utilizando-se o conjunto placa/amostra ilustrado na Fig. 4(b). Foram avaliados parâmetros que podem influenciar as dimensões dos entalhes como: profundidade, largura e raio de curvatura da ponta do entalhe. Foram usadas lâminas de barbear de 2 espessuras: de $0,06 \mathrm{~mm}$ e $0,21 \mathrm{~mm}$ (Fig. 5) e pastas de diamante com várias granulometrias, conforme item 1.2.

\section{RESULTADOS E DISCUSSÃO}

A Fig. 8 ilustra uma placa com sete amostras entalhadas com lâmina de barbear e pasta de diamante.

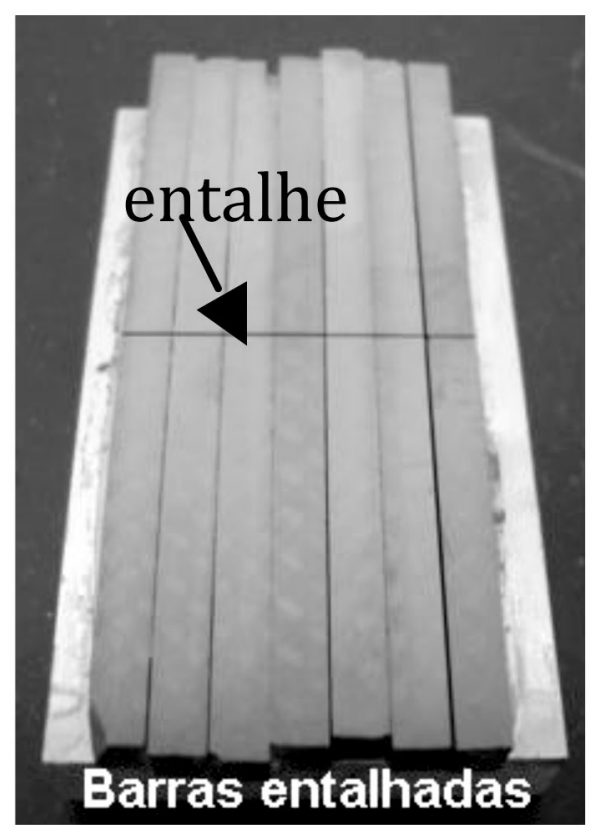

Figura 8: Conjunto amostras/placa suporte, mostrando o entalhe já executado nas amostras.

[Figure 8: Setup of the specimens and support-plate showing the notch already done.]

\subsection{Efeito da granulometria das pastas de diamante no} entalhe

A Fig. 9 mostra os entalhes realizados nas amostras de $\mathrm{SiC}+10 \%\left(\mathrm{Al}_{2} \mathrm{O}_{3}+\mathrm{Yb}_{2} \mathrm{O}_{3}\right)$ pela ação da lâmina de barbear e das pastas de diamante pelo tempo de $1 \mathrm{~h}$. As pastas usadas tinham as granulometrias indicadas nas imagens $(0,25$ até $15 \mu \mathrm{m})$. Todas as pastas causaram o entalhamento, sendo que houve pequenos incrementos na profundidade do entalhe da pasta de $0,25 \mu \mathrm{m}$ para a de $1 \mu \mathrm{m}$ e dessa para a de $3 \mu \mathrm{m}$, o que não foi observado com a mudança da pasta de $3 \mu \mathrm{m}$ para a de $6 \mu \mathrm{m}$, que praticamente não apresentaram diferenças na profundidade. Com a pasta de $6 \mu \mathrm{m}$ o entalhe ficou um pouco mais largo. Já as pastas de $9 \mu \mathrm{m}$ e $15 \mu \mathrm{m}$ produziram entalhes com menores profundidades e mais largos em relação às de 3 e $6 \mu \mathrm{m}$. Todas as medidas de profundidade $(\mathrm{P})$ e de raio da ponta a do entalhe $(\mathrm{R})$, se encontram marcadas nas respectivas amostras da Fig. 9. Para cada entalhe foi usada uma lâmina nova para evitar o efeito do seu desgaste e até mesmo amassamento de seu fio durante o entalhamento.

Este teste foi repetido várias vezes com o objetivo de se verificar a reprodutibilidade do trabalho de entalhe. Não foram colocadas imagens dos outros testes por serem muito semelhantes às apresentadas na Fig. 9, o que indicou ótima reprodutibilidade. Assim, pode-se escolher para fazer o entalhe preliminar do $\mathrm{SiC}$ tanto a pasta de $3 \mu \mathrm{m}$ quanto a 


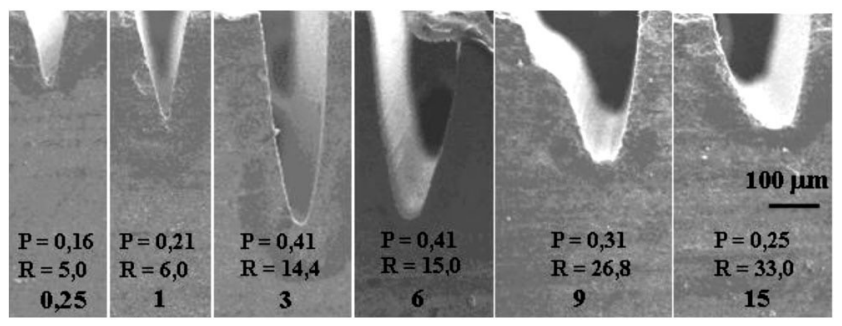

Figura 9: Entalhes obtidos com lâmina de barbear fina e pastas de diamante de 0,$25 ; 1 ; 3 ; 6 ; 9$ e $15 \mu \mathrm{m}$. P (mm) é a medida da profundidade e $\mathrm{R}(\mu \mathrm{m})$ é o raio de curvatura da ponta do entalhe. [Figure 9: Notches produced by the fine razor blade and graphite paste of $0.25 ; 1 ; 3 ; 6 ; 9$ and $15 \mu \mathrm{m}$ in size. $P(\mathrm{~mm})$ is the length of the notch and $R(\mu \mathrm{m})$ is the radius of notch tip.]

de $6 \mu \mathrm{m}$, pois a espessura e profundidade são parecidas e os preços das mesmas são iguais.

\subsection{Efeito da espessura da lâmina no entalhe}

Neste item o objetivo foi verificar o raio do entalhe em função da espessura da lâmina usada para produzir o entalhamento. Foi usada pasta de $6 \mu \mathrm{m}$ e lâmina grossa e fina. O tempo de entalhamento para ambas as lâminas foi de $1 \mathrm{~h}$.

A Fig. 10 mostra os entalhes feitos com as lâminas fina (a e b) e com a lâmina grossa (c e d). Verifica-se que para o mesmo tempo de entalhamento, a lâmina fina produz uma maior profundidade (Fig. 10a) em relação ao entalhe produzido pela lâmina grossa. Também se verifica que o raio de curvatura produzido pela lâmina fina é menor (b) que o produzido pela lâmina grossa $(\mathrm{d})$.

Observa-se uma grande diferença no raio de curvatura (R) da ponta dos entalhes, indicando a facilidade de se obter entalhes com várias espessuras dependendo somente da espessura da lâmina a ser usada. Isso é muito importante, uma vez que se pode eliminar a necessidade do entalhe inicial grosso utilizando-se uma retificadora e disco de corte, cujo procedimento pode trazer consequiências indesejáveis para a amostra a ser analisada, como por exemplo, a geração de microtrincas na ponta do entalhe que vão concentrar tensões e levar a resultados imprecisos de $\mathrm{K}_{\mathrm{IC}}$. Essa sistemática de entalhar inicialmente com disco de corte é praticada pela maioria dos usuários da técnica SEVNB e está indicada também na norma ISO 23146:2008.

\subsection{Entalhamento em etapas com pastas diamantadas de} $6 \mu \mathrm{m}$ e $1 \mu \mathrm{m}$

Com os resultados obtidos anteriormente, decidiu-se fazer um entalhamento por etapas, tomando-se a pasta de $6 \mu \mathrm{m}$ para a produção do entalhe preliminar, e depois um afinamento com a pasta de $1 \mu \mathrm{m}$. A profundidade dos entalhes foram controladas pelo micrômetro da máquina, que foi programado antes dos testes.

A escolha dessas duas pastas foi a partir dos resultados mostrados nas Figs. 9 e 10, em que se pode observar que a pasta de $6 \mu \mathrm{m}$ promove entalhe com maior profundidade e

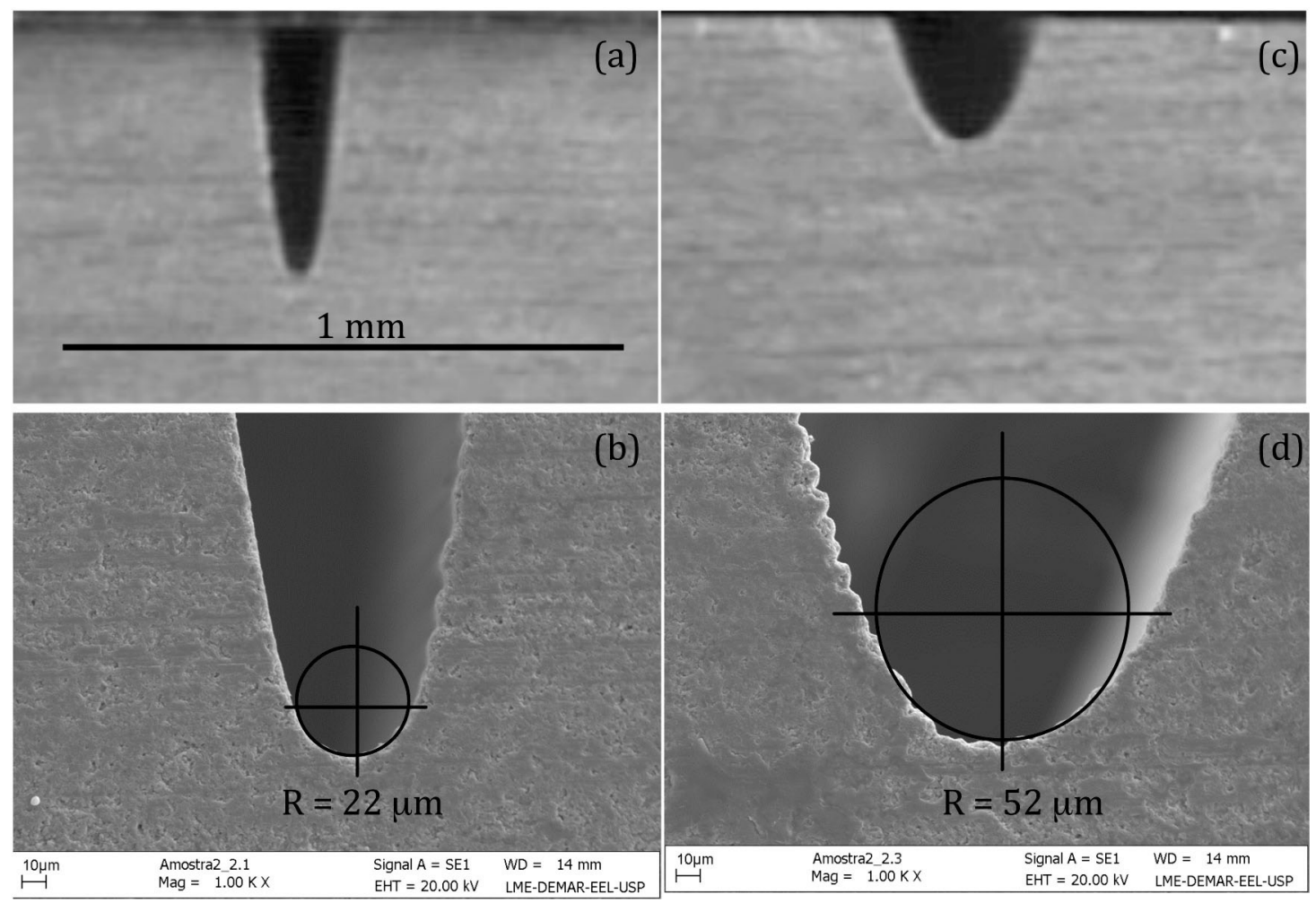

Figura 10: Fotografias obtidas com lupa estereoscópica (a e c) e micrografias obtidas por MEV (b e d) dos entalhes produzidos com pasta de 6 um e lâminas (a e b) fina e (c e d) grossa.

[Figure 10: Stereomicroscope-pictures ( $a$ and $c$ ) and SEM micrograph ( $b$ and $d$ ) of the notch produce by diamond paste of $6 \mu \mathrm{m}$ particle size and the fine razor blade $(b)$ and thick razor blade $(d)$. 

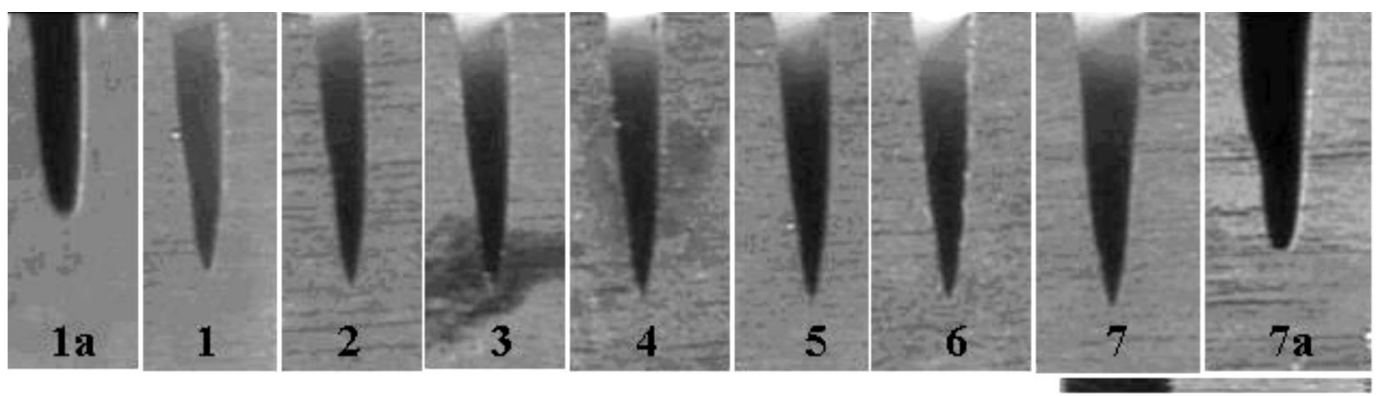

Figura 11: Entalhes realizados com pasta de diamante de $6 \mu \mathrm{m}$ (1a e 7a) e entalhes com pasta de $6 \mu \mathrm{m}$ e afinados com $1 \mu \mathrm{m}$ (1 a 7). Para ambas as pastas foi usada a lâmina fina. A barra de medida é de $1 \mathrm{~mm}$.

[Figure 11: Notches produced using $6 \mu \mathrm{m}$ diamond paste (1a and 7a) and notches produced using $6 \mu \mathrm{m}$ diamond paste followed by polishing using $1 \mu \mathrm{m}$ diamond paste (1 to 7). Both diamond pastes were used in combination with the fine razor blade. The bar is $1 \mathrm{~mm}$ in length.]

Tabela I - Profundidade e espessura dos entalhes mostrados na Fig. 11.

[Table I - Depth and thickness of the notches showed in Fig. 11.]

\begin{tabular}{cccccccccc}
\hline Amostra & $1^{\mathrm{a}}$ & 1 & 2 & 3 & 4 & 5 & 6 & 7 & $7 \mathrm{a}$ \\
\hline Pasta $(\mu \mathrm{m})$ & 6 & $6-1$ & $6-1$ & $6-1$ & $6-1$ & $6-1$ & $6-1$ & $6-1$ & 6 \\
Espessura $(\mathrm{mm})$ & 0,15 & 0,15 & 0,15 & 0,16 & 0,18 & 0,18 & 0,18 & 0,22 & 0,22 \\
Profundidade $(\mathrm{mm})$ & 0,66 & 0,84 & 0,87 & 0,89 & 0,90 & 0,91 & 0,92 & 0,94 & 0,76 \\
\hline
\end{tabular}

ligeiramente mais largo que a de $3 \mu \mathrm{m}$. O uso da pasta de 1 $\mu \mathrm{m}$ para a finalização do entalhe está justificado pela geração de um entalhe com um pequeno raio de curvatura na sua ponta, como indicado na Fig. 9.

Foram realizados dois entalhamentos, adotando-se os mesmos padrões, cujos resultados foram idênticos e, por isso, serão mostradas fotografias somente de um dos testes. A Fig. 11 mostra os corpos entalhados com lâmina fina e pastas de 6 e $1 \mu \mathrm{m}$. Os números colocados nas amostras significam suas respectivas posições na placa para entalhamento, sendo la e $7 \mathrm{a}$ as amostras entalhadas somente com a pasta de $6 \mu \mathrm{m}$ e as demais foram entalhadas com pasta de $6 \mu \mathrm{m}$ inicialmente e posteriormente afinadas com pasta de $1 \mu \mathrm{m}$.

Observa-se que a profundidade e a espessura dos entalhes são um pouco diferentes ao longo das amostras entalhadas em uma mesma placa. A Tabela I mostra as dimensões dos respectivos entalhes. Isso supostamente é devido à distribuição da alimentação da pasta de diamante que é mais efetiva no lado em que se encontra a amostra 7 tendo um maior volume de abrasivo nessa região e causando maior desgaste no material. Isso não implica em deficiência do método, pois a própria norma prevê uma variação de 0,8 a $1,2 \mathrm{~mm}$ na profundidade do entalhe, sendo que essa diferença não afeta os resultados.

Esses testes mostraram que apesar do uso do mesmo material e as mesmas condições de operação da máquina de entalhe, os resultados ainda podem ser ligeiramente diferentes, pois o raio de curvatura médio para os entalhes ficou entre 5,58 e $7,36 \mu \mathrm{m}$.

\subsection{Entalhamento em etapas com pastas diamantadas} de 6,1 e $0,25 \mu \mathrm{m}$

Nessa etapa do trabalho as amostras foram entalhadas
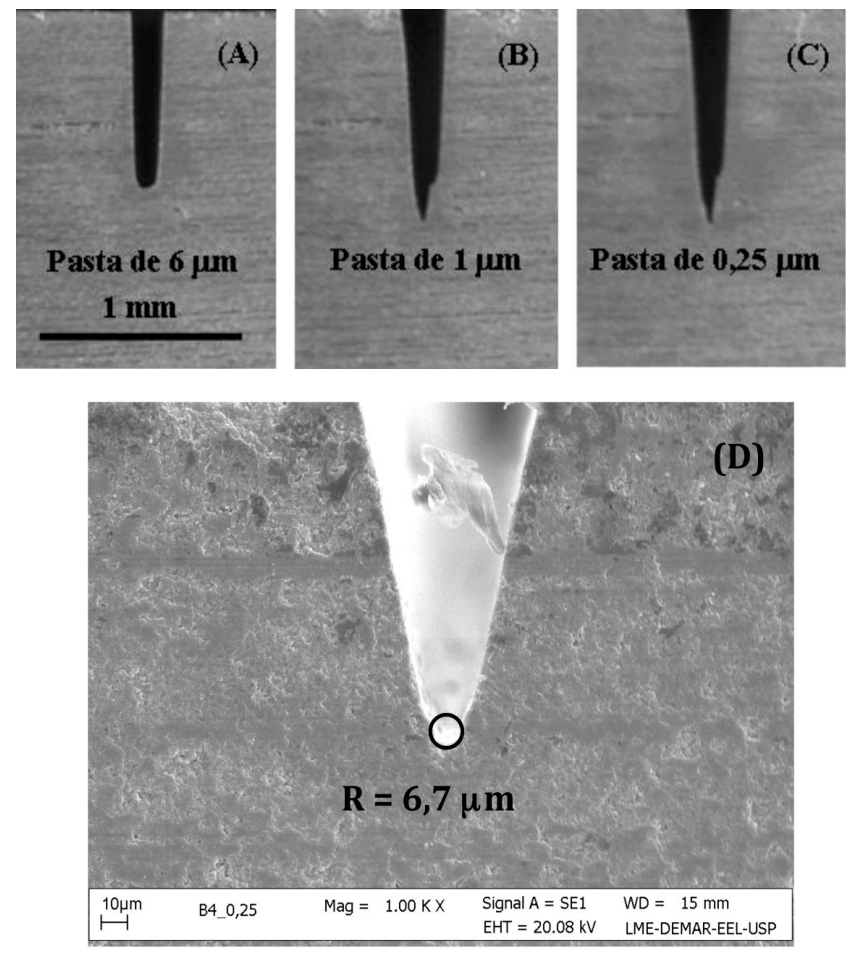

Figura 12: Fotografias produzidas por lupa estereoscópica dos entalhes feitos com as pastas de 6,1 e $0,25 \mu \mathrm{m}$, mostrando o afinamento seqüencial em função da granulometria da pasta usada. (A, B e C) e (D) imagem obtida por MEV da ponta do entalhe final produzido com a pasta final de $0,25 \mu \mathrm{m}$.

[Figure 12: Stereoscope-pictures of the notch produced using diamond past of 6, 1 and $0.25 \mu \mathrm{m}$ in particle size, showing the sequential polishing as a function of the particle size of the diamond pastes ( $A, B$ and $C$ ) and SEM pictures of the final notch tip produced using the $0.25 \mu \mathrm{m}$ paste $(D)$.] 
em etapas seqüenciais: primeiramente com pasta de $6 \mu \mathrm{m}$, na seqüência com a de $1 \mu \mathrm{m}$ e por fim com a de 0,25 $\mu \mathrm{m}$, todas com a lâmina fina. Após cada etapa de entalhamento, as amostras foram lavadas com água e detergente para retirada dos resíduos da pasta anterior.

A Fig. 12 ilustra os entalhes produzidos com as respectivas pastas. Para compor essa figura foram escolhidas somente as amostras da posição 1 na placa mostrada na Fig. 4. As inscrições nas fotografias "pasta de $6 \mu \mathrm{m}$ " e demais indicam as pastas usadas para o afinamento final do entalhe.

Um fato importante que foi verificado é que quando se faz o entalhamento direto com pasta de uma única granulometria há um travamento da lâmina, dificultando o aprofundamento do entalhe.

A Fig. 13 ilustra as superfícies de fratura e do entalhe de uma amostra entalhada, após ruptura sob flexão em que se observa a área fraturada e a área entalhada. De acordo com a norma ISO 23146:2008 a profundidade do entalhe deve estar entre 0,8 e 1,2 $\mathrm{mm}$ que somando com a fratura deve dar aproximadamente $3 \mathrm{~mm}$.

Amostras produzidas com $\mathrm{SiC}$ aditivado com $10 \%$ em volume de $\mathrm{Al}_{2} \mathrm{O}_{3}+\mathrm{Yb}_{2} \mathrm{O}_{3}$ sinterizadas a $1950{ }^{\circ} \mathrm{C}$ e entalhadas com lâmina fina e inicialmente com pasta de diamante de $6 \mu \mathrm{m}$ e finalmente com pasta de 0,25 $\mu \mathrm{m}$, apresentaram valores de $\mathrm{K}_{\mathrm{IC}}$ de 3,43 $\pm 0,23 \mathrm{MPa} \cdot \mathrm{m}^{1 / 2}$. Esse valor está compatível com o encontrado na literatura para cerâmicas de $\mathrm{SiC}$ aditivadas com alguns óxidos de terras raras, por exemplo $\mathrm{SiC}+\left(\mathrm{AlN}+\mathrm{Y}_{2} \mathrm{O}_{3}\right)$ produziram resultados da ordem de $4 \mathrm{MPa} \cdot \mathrm{M}^{1 / 2(1,4)}$.

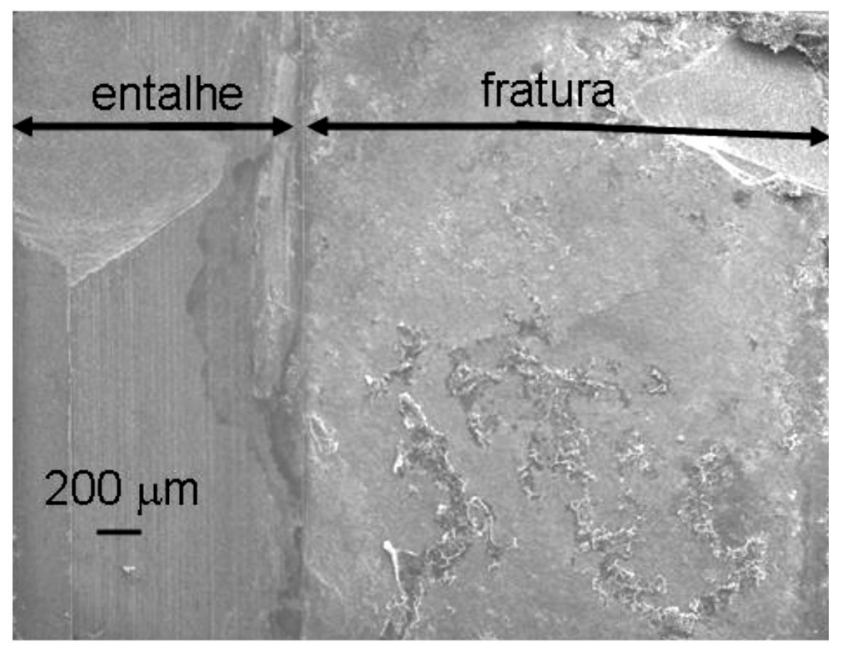

Figura 13: Micrografia obtida por MEV de uma amostra de SiC após ruptura, para determinação de $\mathrm{K}_{\mathrm{IC}}$ pelo método SEVNB, mostrando as seções de fratura e de entalhe.

[Figure 13: SEM picture of a SiC sample after rupture during the measurement of the $K_{I C}$ applying the SEVNB method. The picture shows the fractured part and the notch part of the surface.]

\section{CONCLUSÕES}

As dimensões dos entalhes, necessários para a medida de $\mathrm{K}_{\mathrm{IC}}$ pelo método $\mathrm{SEVNB}$, podem ser produzidas conforme a necessidade, pois com o uso de lâminas de barbear com dimensões adequadas e pastas com granulometrias específicas, isso é perfeitamente possível.

O entalhamento deve ser feito em no mínimo duas etapas, pois com o uso de uma única pasta, à medida que o entalhe vai se tornando mais profundo aumenta-se a superfície da lâmina em contato com as paredes do entalhe, o que pode causar travamento da máquina. Se isso ocorrer, pode resultar em dano à máquina e defeitos nos entalhes.

Conseguiu-se obter entalhes com raio de curvatura de suas pontas da ordem de $6 \mu \mathrm{m}$. Isso valida a técnica SEVNB para $\mathrm{SiC}$, pois normalmente essas cerâmicas apresentam tamanhos médios de grão em torno de $10 \mu \mathrm{m}$, satisfazendo a relação $\mathrm{R}<2 \mathrm{~d}$ citada na revisão bibliográfica.

A justificativa de não se utilizar o método SEVNB para a determinação de $\mathrm{K}_{\mathrm{IC}}$, devido a dificuldade de se produzir o entalhe em "V", com raios de curvatura e tamanhos adequados, fica resolvida, pois este trabalho mostra que isso é perfeitamente viável, desde que se tenha o equipamento e procedimentos adequados.

$\mathrm{O} \mathrm{SiC}+10 \%\left(\mathrm{Al}_{2} \mathrm{O}_{3}+\mathrm{Yb}_{2} \mathrm{O}_{3}\right)$ preparado neste trabalho apresentou o valor de $\mathrm{K}_{\mathrm{IC}}$, determinado pela metodologia aqui descrita, igual a 3,43 $\pm 0,23 \mathrm{MPa} . \mathrm{m}^{1 / 2}$, que está dentro da faixa de valores encontrada na literatura para materiais similares a esse.

\section{AGRADECIMENTOS}

Os autores agradecem a FAPESP (Proc. 2010/51925-6) ao CNPq pelas bolsas de produtividade (Procs. 303061/20090 e 304760/2010-2, à CAPES pelas bolsas de mestrado e doutorado atribuídas ao Programa de Pós-Graduação em Engenharia de Materiais da EEL.

\section{REFERÊNCIAS}

[1] K. Strecker, S. Ribeiro, R. Oberacker, M. J. Hoffmann, Int. J. Refractory Mater. Hard Mater. 22 (2004) 169-175.

[2] R. Damani, R. Gstrein, R. Danzer, J. Eur. Ceram. Soc. 16 (1996) 695-702.

[3] H. Awaji, T. Watanabe, Y. Sakaid, H. Nakagawa, Ceram. Int. 18 (1992) 1-17.

[4] K. Strecker, S. Ribeiro, M. J. Hoffmann, Mater. Res. 8 (2005) p.121-124.

[5] S. S. Scherrer, I. L. Denry, H. W. A.Wiskott, Dent. Mater. 14 (1998) 246-255.

[6] J. M. Calderon-Moreno, M. Popa, Mater. Sci. Eng. A 319-321 (2001) 692-696.

[7] L. A. Santos, R. C. Souza, C. Santos, K. Strecker, F. A. Santos, S. Ribeiro, R. Oberacker, Anais $64^{\circ}$ Cong. ABM, Belo Horizonte, MG (2009) 223.

[8] R. J. Damani, C. Schster, R. Danzer, J. Eur. Ceram. Soc. 17 (1997) 1685-1689.

[9] S. R. Choi, J. P. Gyekenyesi, "Assessments of fracture toughness of monolithic ceramics - SEPB versus SEVNB methods", $11^{\text {th }}$ Int. Conf. Fracture, ICF, ESIS, and ASTM, Turin, Italy, March 20-25, 2005 - Publicação NASA National Aeronautics and Space Administration (2006). 
[10] H. Wang, P. Pallav, G. Isgo, A. Feizer, Dent. Mater. 23 (2007) 905-910.

[11] G. A. Gogotsi, Ceram. Int. 29 (2003) 777-784.

[12] ASTM C 1421- 09, Standard test method for determination of fracture toughness of advanced ceramics at ambient temperature (2009) 32p.

[13] G. D. Quinn, The fracture toughness round robins in VAMA: What we have learned - Fracture resistance testing monolithic and composite brittle materials, ASTM 1409, Am. Soc. for Testing and Materials, West Conshohocken, PA, (2002).

[14] ISO 23146:2008, Fine ceramics (advanced ceramics, advanced technical ceramics) - Test methods for fracture toughness of monolithic ceramics - Single-edge V-notch beam (SEVNB) method (2008).

[15] H. Özcoban, H. Jelito, G. A. Schneider, J. Eur. Ceram. Soc. 30 (2010) 1579-1583.
[16] G. D. Quinn, R. C. Bradt, J. Am. Ceram. Soc. 90 (2007) 673-680.

[17] D. Carolan, P. Alveen, C. A. Ivankovi, N. Murphy, Eng. Fract. Mec. 78 (2011) 2885-2895.

[18] H. Fischer, A. Waindich, R. Telle, Dent. Mater. 24 (2008) 618-622.

[19] J. J. Kübler, Fracture toughness of ceramics using the SEVNB method: From preliminary study to a Standard Test Method, Fracture testing of monolithic and composite brittle materials, ASTM STP 1409, Eds. Am. Soc. Testing Mater., West Conshohocken, PA, EUA (2002).

[20] Int. Org. Stand., ISO 23146:2008, Fine ceramics (advanced ceramics, advanced technical ceramics) - Test methods for fracture toughness of monolithic ceramics Single-edge V-notch beam (SEVNB) method, Geneva (2008) $16 \mathrm{p}$.

[21] B. Atzori, P. Lazarrin, Int. J. Fract. 107 (2001) 3-8.

(Rec. 25/09/2012, Ac. 10/11/2012) 